\title{
Association Between Triglyceride-Glucose Index and 2-Year Adverse Cardiovascular and Cerebrovascular Events in Patients with Type 2 Diabetes Mellitus Who Underwent Off-Pump Coronary Artery Bypass Grafting
}

\author{
Liang Chen, Xiao-Hang Ding, Kang-Jun Fan, Ming-Xin Gao, Wen-Yuan Yu, Hong-Li Liu, Yang Yu (D) \\ Department of Cardiac Surgery, Beijing Anzhen Hospital, Capital Medical University, Beijing, 100029, People's Republic of China \\ Correspondence: Yang Yu, Email heartyuyang@hotmail.com
}

Background: Data on the relationship between the triglyceride glucose (TyG) index and prognosis after off-pump coronary artery bypass grafting (OPCABG) are limited. This retrospective observational cohort study evaluated the association of the TyG index with prognosis in patients with diabetes mellitus who underwent OPCABG.

Methods: The TyG index was calculated using the following equation: TyG index $=\ln$ (fasting triglyceride level [mg/dL] $\times$ fasting glucose level $[\mathrm{mg} / \mathrm{dL}] / 2)$. The primary outcomes included the occurrence of major adverse cardiovascular and cerebrovascular events (MACCEs), which were defined as all-cause death, nonfatal myocardial infarction, nonfatal stroke and symptomatic graft failure. The association between the TyG index and MACCEs was assessed by Cox proportional hazards regression analysis.

Results: A total of 1578 patients with diabetes who underwent OPCABG (mean age, 62.9 \pm 8.0 years; men, 72.7\%) were enrolled in this study. Over the follow-up of 2 years, 176 patients (11.2\%) had at least 1 primary endpoint event. The follow-up incidence of the primary endpoint rose with increasing TyG index tertiles. The multivariate Cox proportional hazards regression analysis adjusted for multiple confounders revealed a hazard ratio for the primary endpoint of 2.133 (95\% CI 1.347-3.377; P for trend $=0.001)$ when the highest and lowest TyG index tertiles were compared.

Conclusion: The TyG index was significantly and positively associated with MACCEs, suggesting that the TyG index may be a valuable predictor of adverse cardiovascular and cerebrovascular outcomes after OPCABG in patients with T2DM.

Keywords: triglyceride-glucose index, off-pump coronary artery bypass grafting, major adverse cardiovascular and cerebrovascular events, type 2 diabetes mellitus

\section{Introduction}

Coronary artery bypass grafting $(\mathrm{CABG})$ remains the preferred treatment option for patients with severe multiple vessel coronary artery disease. ${ }^{1}$ In the past two decades, the proportion of coronary artery bypass grafting (CABG) has been rising fast, more than half of which is off-pump CABG (OPCABG) in mainland China. ${ }^{2}$ Although surgical techniques and medical treatment have been developed, the risk of adverse cardiovascular and cerebrovascular events (MACCEs) after OPCABG remains relatively high. ${ }^{3}$ The triglyceride-glucose (TyG) index may be a useful marker for prognosis after OPCABG.

TyG index, a product of triglycerides and fasting plasma glucose (FPG), is a good tool that correlates with surrogate and direct measures of insulin resistance (IR). ${ }^{4,5}$ Previous studies have reported that the TyG index is significantly associated with an increased risk of developing T2DM, hypertension and adverse cardiovascular events. ${ }^{6-9}$ Moreover, several cross-sectional studies have reported a strong relation between the TyG index and atherosclerosis in different 
clinical conditions. ${ }^{10-12}$ However, whether TyG may be associated with MACCEs of patients with T2DM who were treated with OPCABG has not yet been assessed. Therefore, in the present study, we examined the relationship between the baseline TyG index and MACCE outcomes in 1578 Chinese patients with T2DM who underwent OPCABG.

\section{Methods}

\section{Study Population and Design}

The present study is a single-center, observational, retrospective cohort study among patients with type 2 diabetes who underwent isolated OPCABG surgery at Beijing Anzhen Hospital between September 2017 to June 2019. We finally enrolled 1578 patients in the present study. In these patients, 4576 saphenous vein grafts and 1125 arterial grafts $(97.8 \%$ LIMA, 2.2\% bilateral internal mammary artery) were implanted. All patients undergoing concomitant surgery (eg, valve, vascular, or congenital) and patients undergoing re-do CABG were excluded. All patients with perioperative death, cardiogenic shock, extreme body mass index $\left(B M I>45 \mathrm{~kg} / \mathrm{m}^{2}\right.$ ), suspected familial hypertriglyceridemia [plasma triglycerides $\geq 500 \mathrm{mg} / \mathrm{dL}(5.65 \mathrm{mmol} / \mathrm{L})$ or more than one first-degree relative with triglycerides $\geq 500 \mathrm{mg} / \mathrm{dL}$ ] were also excluded. Twelve patients were also excluded because of missing follow-up data despite at least 3 separate attempts to contact them (Figure 1). This study was performed in accordance with the Helsinki Declaration of Human Rights and approved by the institutional review board of Beijing Anzhen Hospital, Capital Medical University. Given the retrospective nature of the present research, no informed consent was required. All patients' identifiable information were hidden and anyone's identity cannot be deduced from the context.

\section{Data Collection}

Data on demographics, personal medical history, and medication history, such as age, sex, BMI, current smoking status, current drinking status et al, were collected using a standard questionnaire. The blood pressure on admission was recorded. Body mass index (BMI) was calculated using the following equation: BMI $=$ weight $(\mathrm{kg}) / \mathrm{height}\left(\mathrm{m}^{2}\right)$. Blood samples were taken after overnight fasting ( $>8 \mathrm{~h}$ ). Serum levels of fasting plasma glucose (FPG), glycated albumin (GA) and lipid profiles, including triglyceride (TG), total cholesterol (TC) and high-density lipoprotein cholesterol (HDL-C), were determined by standard laboratory techniques. The low-density lipoprotein cholesterol (LDL-C) level was computed with the Friedewald equation. The TyG index was computed using the following formula: TyG index $=\ln$ [fasting $\mathrm{TG}(\mathrm{mg} / \mathrm{dL}) \times \mathrm{FPG}(\mathrm{mg} / \mathrm{dL}) / 2] . \mathrm{CrCl}<60 \mathrm{~mL} / \mathrm{min}$ was considered the criterion for chronic kidney disease (CKD). $\mathrm{CrCl}$ was calculated using the Cockcroft and Gault formula. ${ }^{13}$ Left ventricular ejection fraction (LVEF) was evaluated by a two-dimensional modified Simpson's method using an ultrasonic cardiogram (Philips Company, Eindhoven, The Netherlands). Patients with signs/symptoms of congestive heart failure (CHF), current treatment for CHF, or objective evidence of reduced ejection fraction (LVEF $<40 \%$ ) were considered to have cardiac failure. The "use of arterial graft" means that at least one arterial conduit is used in $\mathrm{CABG}$, which can be left internal mammary artery (LIMA), right internal mammary artery (RIMA) or radial artery (RA).

\section{Endpoints}

All patients were followed up at every 6 months after hospital discharge. The primary observational endpoint was defined as MACCE consisting of four events within 2 years after OPCABG as follows: all-cause death, nonfatal myocardial infarction (MI), nonfatal stroke and symptomatic graft failure. The secondary observational endpoint was each component of the composite primary endpoint. MI was defined as elevated cardiac troponin higher than the upper reference limit with ischemia indicated by symptoms and/or electrocardiographic changes with or without an elevation of the ST segment. The presence of new pathological Q waves in $\geq 2$ contiguous electrocardiogram leads was also diagnosed as MI. Symptomatic graft failure was defined as having typical angina symptoms again after discharge and identification of graft occlusion or stenosis of $\geq 70 \%$ by coronary CTA scan. All patients having typical angina symptoms after OPCABG underwent CTA scan, some of which underwent further PCI. The first primary endpoint event that occurred during the follow-up was used for analysis in the current study. For patients with multiple adverse outcomes occurring almost simultaneously during the follow-up, only the most severe event (all-cause death $>$ nonfatal MI $>$ nonfatal stroke $>$ 


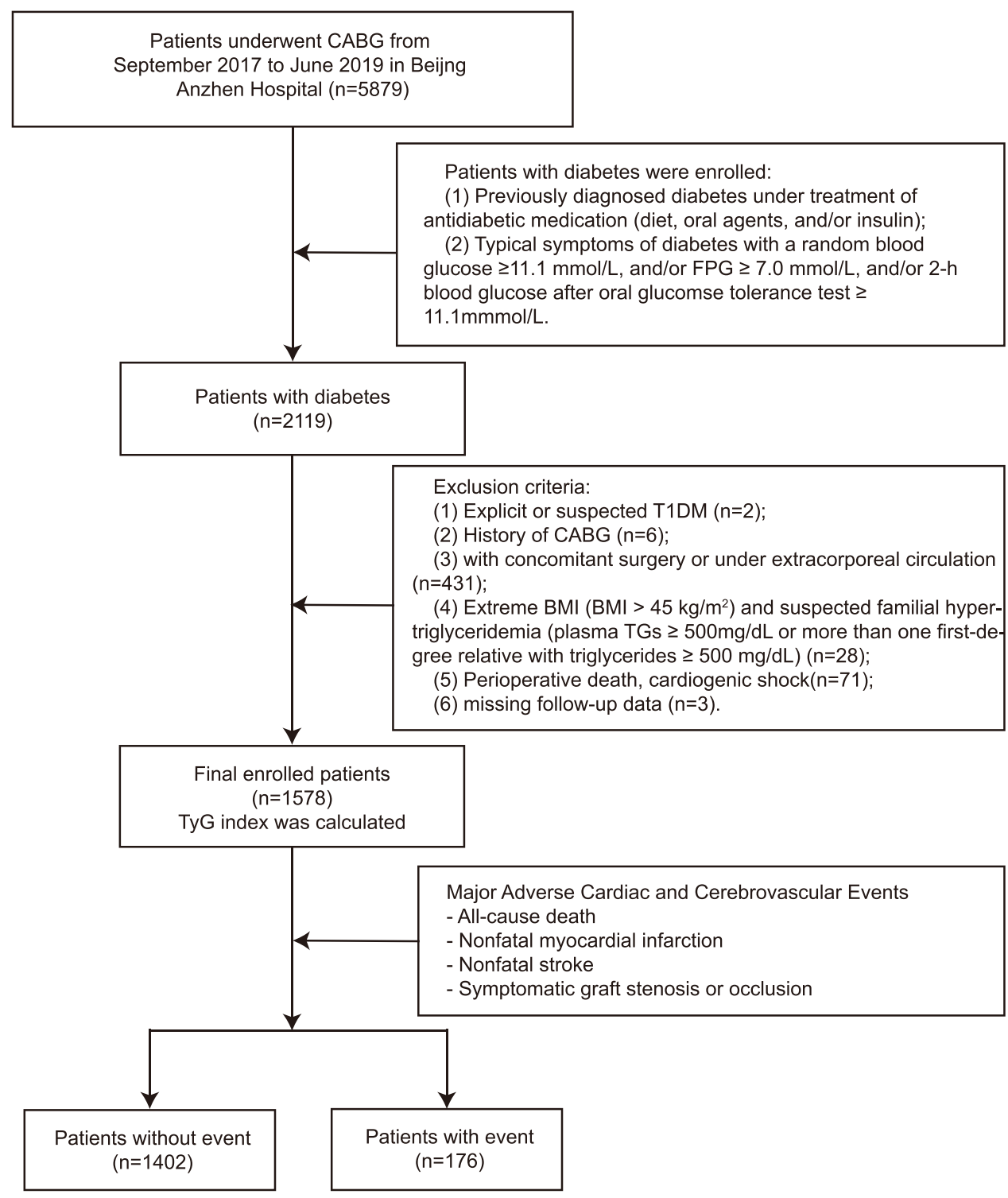

Figure I Flow chart of the study population enrollment.

Abbreviations: CABG, coronary artery bypass graft; FPG, fasting plasma glucose; TIDM, type I diabetes mellitus; TGs, triglycerides; BMI, body mass index; TyG, triglyceride glucose.

symptomatic graft failure) was selected for the analyses. If the same event occurred multiple times, only the first occurrence was used for the analyses.

\section{Statistical Analysis}

Continuous variables are presented as the mean \pm standard deviation (SD) or median (25th and 75th percentiles: P25, P75) in the case of normal or nonnormal distribution. All patients were stratified into three groups (T1 [TyG index < 8.850], T2 [8.850 $\leq$ TyG index <9.384], and T3 [TyG index $\geq 9.384]$ ]) in accordance with tertiles of the TyG index. The Chi squared test or Fisher's exact test was used to analyze differences in categorical variables between groups. ANOVA or the Kruskal-Wallis $H$-test was applied to analyze differences in continuous variables between groups. Kaplan-Meier curves and Log rank tests were used to determine statistically significant survival differences among these three groups of patients. We conducted a Cox proportional hazards regression analysis to estimate the hazard ratios (HRs) and their $95 \%$ confidence intervals (CIs) of developing the primary and second endpoints. In the Cox models, each continuous variable's HR indicates that the risk of the primary endpoint change if the variable increase per unit. The TyG index 
was analyzed in two ways: 1) as a categorical variable and 2) as a continuous variable. Predictors of the incidence of the primary endpoint identified through univariate analysis were also tested in a multivariate analysis. In the multivariate model, the following confounders were chosen because of their clinical importance and statistical significance in the univariate analysis: age (continuous), sex, BMI (continuous), current smoking, hypertension, previous MI, previous stroke, past PCI, cardiac failure, CKD, preoperative LVEF, insulin dependence, LDL-C (continuous), HDL-C (continuous), diagnosis, extent of CAD, left main disease, complete revascularization, and use of IABP. Statistical analyses were performed using SPSS version 24.0 software (IBM Corporation, Chicago, IL) and the R Programming Language (version 4.0.5), and $\mathrm{P}<0.05$ was considered statistically significant.

\section{Results}

\section{Baseline Characteristics}

The study patients had an average age of $62.9 \pm 8.0$ years and $1116(70.7 \%)$ patients were male. The mean levels of TyG index of the three groups were $8.45 \pm 0.31,9.11 \pm 0.15$ and $9.83 \pm 0.34$, respectively. The baseline clinical and laboratory characteristics of the study patients according to the TyG index tertiles are presented in Table 1. Patients with a high TyG index were more likely to have a history of dyslipidemia and stroke. BMI, TC level, LDL-C level, FPG level and triglyceride level increased, whereas HDL-C level decreased in proportion to the TyG index tertiles. The proportion of current smokers and insulin dependence significantly increased with an increase in the TyG index. And the proportion of acute myocardial infarction and 3-vessel disease also increased with an increase in the TyG index. And no significant difference was found in the other indicators. Patient characteristics, periprocedural and angiographic findings, and procedural results of the study patients according to the TyG index tertiles are listed in Table 1.

\section{TyG Index and Endpoints}

During the 2-year follow-up, 176 patients $(11.2 \%)$ had at least 1 primary endpoint event, which was recorded in 29 (5.5\%) patients from the T1 group, 59 (11.2\%) from the T2 group, and 88 (16.7\%) from the T3 group. In the 176 patients who had at least 1 primary endpoint event, there were 17 deaths ( 9 deaths from cardiovascular causes, 3 deaths from stroke and 3 deaths from other causes), 39 cases of nonfatal strokes, 40 cases of nonfatal MI, and 79 cases of symptomatic graft failure. The respective incidences of all-cause death, nonfatal stroke, nonfatal MI and symptomatic graft failure among the TyG index tertiles are shown Table 2.

Table 2 shows the Cox proportional hazard analysis of TyG tertiles and 2-year event rate for all-cause death, nonfatal MI, nonfatal stroke, symptomatic graft failure and MACCE. On unadjusted Cox modeling, the rate of nonfatal MI, nonfatal stroke, symptomatic graft failure and MACCE rose significantly with elevated TyG index levels $(\mathrm{P}<0.05$ for trend). However all-cause death across three groups has no difference. The rate of MACCE, nonfatal MI and symptomatic graft failure increased with rising TyG index levels after adjusting for age, sex, BMI, current smoking, hypertension, previous MI, previous stroke, past PCI, cardiac failure, CKD, preoperative LVEF, insulin dependence, LDL-c, HDL-C, diagnosis, extent of CAD, left main disease, complete revascularization, and use of IABP. Whereas all-cause death and nonfatal stroke have no difference across the three groups.

Kaplan-Meier curves of the incidence of the primary endpoint and each component event of the primary endpoint for the TyG index tertiles are presented in Figure 2. The incidence of the primary endpoint in the T3 group was significantly higher than that in the T1 group (P log-rank $<0.0001$ ). This difference was mainly driven by the increase in nonfatal MI (Log rank test, $\mathrm{P}=0.0047$ ), nonfatal stroke (Log rank test, $\mathrm{P}=0.0190)$ and symptomatic graft failure (Log rank test, $\mathrm{P}=$ 0.0002) across the TyG index tertiles. However, the incidence of overall death (Log rank test, $P=0.9400)$ at follow-up were similar among the TyG index tertiles.

Univariate and multivariate Cox proportional hazards regression analyses and predictors for MACCE are presented in Tables 3 and 4. The TyG index at baseline was significantly related to the incidence of the primary endpoint. In the univariate analysis, the TyG index as a continuous variable was associated with an HR of 2.624 (95\% CI 2.076-3.316; $\mathrm{P}<0.001$ ). Adjustment for multiple confounders did not attenuate the relationship (HR 1.954, 95\% CI 1.508-2.533; P < 0.001) (Table 3). The incidence of the primary endpoint increased monotonically across the tertiles of the TyG index (P 
Table I Baseline Clinical and Laboratory Characteristics and of the Study Patients Stratified by the Tertiles of TyG Index

\begin{tabular}{|c|c|c|c|c|}
\hline & TI $n=526$ & T2 $n=526$ & $T 3 n=526$ & $\mathbf{p}$ \\
\hline TyG index & $8.45(0.31)$ & $9.11(0.15)$ & $9.83(0.34)$ & $<0.001$ \\
\hline Age (years) & $62.6(7.5)$ & $63.0(7.8)$ & $63.2(8.7)$ & 0.434 \\
\hline Sex, male, n (\%) & $365(69.4)$ & $379(72.1)$ & $372(70.7)$ & 0.638 \\
\hline BMI $\left(\mathrm{kg} / \mathrm{m}^{2}\right)$ & $25.17(2.96)$ & $26.09(2.98)$ & $26.65(3.25)$ & $<0.001$ \\
\hline SBP (mmHg) & $129.84(16.17)$ & 131.36 (17.95) & $129.63(15.84)$ & 0.187 \\
\hline DBP (mmHg) & $73.37(10.86)$ & $74.00(10.91)$ & $74.94(10.11)$ & 0.055 \\
\hline Current smoking, n (\%) & $136(25.9)$ & $152(28.9)$ & $164(3 \mid .2)$ & 0.160 \\
\hline Hypertension, n (\%) & $387(73.6)$ & 374 (7I.I) & $372(70.7)$ & 0.536 \\
\hline Dyslipidemia, n (\%) & I55 (29.5) & $160(30.4)$ & $204(38.8)$ & 0.002 \\
\hline Previous MI, n (\%) & $61(11.6)$ & $73(13.9)$ & $94(17.9)$ & 0.014 \\
\hline Previous stroke, n (\%) & $36(6.8)$ & $48(9.1)$ & $65(12.4)$ & 0.009 \\
\hline Past PCI, n (\%) & 115 (2I.9) & $117(22.2)$ & $139(26.4)$ & 0.153 \\
\hline Cardiac failure, n (\%) & $16(3.0)$ & $20(3.8)$ & $22(4.2)$ & 0.606 \\
\hline CKD, n (\%) & $17(3.2)$ & $17(3.2)$ & $31(5.9)$ & 0.043 \\
\hline Preoperative LVEF (\%) & $59.64(7.26)$ & $59.55(7.75)$ & $59.31(7.97)$ & 0.768 \\
\hline Insulin dependence, n (\%) & $127(24.1)$ & I5I (28.7) & $167(31.7)$ & 0.022 \\
\hline TC (mg/dl) & $\mid 30.70[|| \mid 1.70,154.60]$ & $14 \mid .70[123.30,163.50]$ & $153.30[129.90,178.80]$ & $<0.001$ \\
\hline TGs (mg/dl) & $87.80[70.00,104.60]$ & $130.30[108.43,154.08]$ & $202.10[156.90,260.70]$ & $<0.001$ \\
\hline FPG (mg/dl) & $110.30[95.00,125.30]$ & $135.00[1 \mid 8.65,165.55]$ & $179.00[145.33,227.95]$ & $<0.001$ \\
\hline LDL-C (mg/dl) & $75.40[60.30,94.30]$ & $84.10[67.88,102.10]$ & $87.95[69.70,109.80]$ & $<0.001$ \\
\hline HDL-C (mg/dl) & $37.90[33.20,44.80]$ & $36.10[32.50,42.58]$ & $34.40[29.80,39.00]$ & $<0.001$ \\
\hline \multicolumn{5}{|l|}{ Diagnosis } \\
\hline Stable CAD, n (\%) & I (0.2) & $2(0.4)$ & $3(0.6)$ & 0.605 \\
\hline UA, n (\%) & $495(94.1)$ & $485(92.2)$ & $461(87.6)$ & 0.001 \\
\hline STEMI, n (\%) & $19(3.6)$ & $26(4.9)$ & $38(7.2)$ & 0.030 \\
\hline NSTEMI, n (\%) & II (2.I) & $13(2.5)$ & $24(4.6)$ & 0.042 \\
\hline \multicolumn{5}{|l|}{ Extent of CAD } \\
\hline I-vessel disease, n (\%) & $52(9.9)$ & $44(8.4)$ & $35(6.7)$ & 0.164 \\
\hline 2-vessel disease, n (\%) & $94(17.9)$ & $86(16.3)$ & $70(13.3)$ & 0.119 \\
\hline 3-vessel disease, n (\%) & $383(72.8)$ & $396(75.3)$ & $430(81.7)$ & 0.002 \\
\hline Left main disease, $\mathbf{n}(\%)$ & $57(10.8)$ & $55(10.5)$ & $74(14.1)$ & 0.136 \\
\hline Use of arterial graft, n (\%) & $392(74.5)$ & $388(73.8)$ & $374(7 I .1)$ & 0.421 \\
\hline Complete revascularization, $\mathrm{n}$ (\%) & $511(97.1)$ & $509(96.8)$ & $509(96.8)$ & 0.919 \\
\hline Use of IABP, $\mathbf{n}(\%)$ & $17(3.2)$ & $19(3.6)$ & $20(3.8)$ & 0.878 \\
\hline
\end{tabular}

Abbreviations: TyG, triglyceride glucose; BMI, body mass index; SBP, systolic blood pressure; DBP, diastolic blood pressure; MI, myocardial infarction; PCl, percutaneous coronary intervention; CKD, chronic kidney disease; LVEF, left ventricular ejection fraction; TC, total cholesterol; TGs, triglycerides; FPG, fasting plasma glucose; LDL-C, low-density lipoprotein-cholesterol; HDL-C, high-density lipoprotein-cholesterol; CAD, coronary artery disease; UA, unstable angina; STEMI, ST-segment elevation myocardial infarction; NSTEMI, non ST-segment elevation myocardial infarction; IABP, intra-aortic balloon pump.

for trend $\leq 0.001$ ) (Table 4). Taking T1 as the reference, multivariate analysis revealed that the TyG index for T2 and T3 increased the HRs for the incidence of the primary endpoint (T2: HR 1.726, 95\% CI 1.093-2.725; T3: HR 2.133, 95\% CI 1.347-3.377) (Table 4).

Relevant clinical variables like clinical diagnosis (unstable angina vs acute $\mathrm{MI}$ ), age ( $\leq 70$ vs $>70$ years), sex (male vs female), and BMI ( $<28 \mathrm{vs} . \geq 28 \mathrm{~kg} / \mathrm{m}^{2}$ ) were subject to post hoc subgroup analyses for the primary endpoint. When the analysis was stratified by age, we found that a higher TyG index was significantly associated with an increased risk of the primary endpoint in patients aged 70 years and younger (adjusted HR 2.251, 95\% CI 1.333-3.800; $\mathrm{P}=0.002$ ); however, the similar result did not occur in patients aged over 70 years (adjusted HR1.385, 95\% CI 0.488-3.935; P = 0.541). When the analysis was stratified by sex, we found that a higher TyG index was significantly associated with an increased risk of the primary endpoint in male patients (adjusted HR 2.463, 95\% CI 1.325-4.577; P = 0.004); however, the similar result 
Table 2 Baseline TyG Index and Prediction of Cardiovascular and Cerebrovascular Events

\begin{tabular}{|c|c|c|c|c|c|c|}
\hline Endpoint & $\begin{array}{l}\text { Baseline TyG } \\
\text { Index }\end{array}$ & $\begin{array}{l}\text { 2-Year Events, } \\
\text { n (\%) }\end{array}$ & $\begin{array}{l}\text { Unadjusted HR (95\% } \\
\mathrm{CI})\end{array}$ & $P$ value & $\begin{array}{l}\text { Adjusted HR (95\% } \\
\mathrm{Cl})\end{array}$ & $\mathbf{P}$ value \\
\hline \multirow[t]{3}{*}{ All-cause death } & Tertile I & $5(0.95)$ & Ref. & $0.94 I$ & Ref. & 0.730 \\
\hline & Tertile 2 & $6(1.14)$ & $1.2(0.366-3.933)$ & 0.763 & $0.947(0.274-3.272)$ & 0.932 \\
\hline & Tertile 3 & $6(1.14)$ & $1.209(0.369-3.962)$ & 0.754 & $0.61(0.153-2.431)$ & 0.483 \\
\hline$P$ trend & & & 0.757 & & 0.472 & \\
\hline \multirow[t]{3}{*}{ Nonfatal MI } & Tertile I & $4(0.76)$ & Ref. & 0.012 & Ref. & 0.034 \\
\hline & Tertile 2 & $15(2.85)$ & $3.787(|.257-||I .4| 2)$ & 0.018 & $3.47(1.14-10.564)$ & 0.029 \\
\hline & Tertile 3 & $20(3.80)$ & $5.130(1.754-15.01)$ & 0.003 & $4.35 \mid(1.436-13.182)$ & 0.009 \\
\hline$P$ trend & & & 0.002 & & 0.010 & \\
\hline \multirow[t]{3}{*}{ Nonfatal stroke } & Tertile I & $7(1.33)$ & Ref. & 0.026 & Ref. & 0.275 \\
\hline & Tertile 2 & $12(2.28)$ & I.727 (0.68-4.387) & 0.250 & $1.409(0.538-3.691)$ & 0.485 \\
\hline & Tertile 3 & $21(3.99)$ & 3.065 (1.303-7.209) & 0.010 & $2.077(0.8 I-5.326)$ & 0.128 \\
\hline$P$ trend & & & 0.007 & & 0.110 & \\
\hline Symptomatic graft & Tertile I & II (2.09) & Ref. & $<0.001$ & Ref. & 0.011 \\
\hline \multirow[t]{2}{*}{ failure } & Tertile 2 & $28(5.32)$ & $2.579(1.284-5.179)$ & 0.008 & $2.625(1.275-5.405)$ & 0.009 \\
\hline & Tertile 3 & $40(7.60)$ & 3.815 (1.958-7.436) & $<0.001$ & $2.987(1.44-6.195)$ & 0.003 \\
\hline$P$ trend & & & $<0.001$ & & 0.005 & \\
\hline \multirow[t]{3}{*}{ MACCE } & Tertile I & $29(5.5 \mathrm{I})$ & Ref. & $<0.001$ & Ref. & 0.005 \\
\hline & Tertile 2 & 59 (1 I.22) & $2.095(1.343-3.268)$ & 0.001 & $1.726(1.093-2.725)$ & 0.019 \\
\hline & Tertile 3 & $88(16.73)$ & $3.297(2.167-5.016)$ & $<0.001$ & 2.133 (1.347-3.377) & 0.001 \\
\hline P trend & & & $<0.001$ & & 0.005 & \\
\hline
\end{tabular}

Abbreviations: TyG, triglyceride glucose; MI, myocardial infarction; MACCE, major adverse cardiovascular and cerebrovascular events.

did not occur in female patients (adjusted HR 1.683, 95\% CI 0.813-3.483; $\mathrm{P}=0.161$ ). When the analysis was stratified by BMI, we found that a higher TyG index was significantly associated with an increased risk of the primary endpoint in patients with BMI $<28 \mathrm{~kg} / \mathrm{m}^{2}$ (adjusted HR 1.873, 95\% CI 1.116-3.144; $\mathrm{P}=0.018$ ); however, the similar result did not occur in patients with BMI $\geq 28 \mathrm{~kg} / \mathrm{m}^{2}$ (adjusted HR 2.662, 95\% CI $0.873-8.116 ; \mathrm{P}=0.085$ ). When the analysis was stratified by clinical diagnosis, we found that a higher TyG index was significantly associated with an increased risk of the primary endpoint in patients with unstable angina (adjusted HR 1.940, 95\% CI 1.170-3.216; P =0.010); however, the similar result did not occur in acute MI (adjusted HR 2.73, 95\% CI 0.756-9.865; P = 0.125).

\section{Discussion}

The primary finding of the present study was that an increased TyG index was a strong indicator of MACCEs, including all-cause death, nonfatal MI and stroke, and symptomatic graft failure, in patients with T2DM who underwent OPCABG, even after adjustment for confounding risk factors. Compared to participants with a lower TyG index tertile, those with a higher TyG index tertile had an apparently higher incidence of MACCEs, nonfatal MI and symptomatic graft failure.

The TyG index was initially designed as a chronic insulin resistance (IR) marker, ${ }^{5,14}$ and studies even showed that the TyG index may have a better performance on the prediction of IR and atherosclerosis compared with the homeostatic model assessment of IR (HOMA-IR) ${ }^{15,16}$ Insulin resistance (IR) is traditionally measured by the HOMA-IR. ${ }^{17}$ However, it is inconvenient to measure insulin levels to calculate HOMA-IR. Several previous studies have suggested that the value of the TyG index for predicting IR is better than that of HOMA-IR ${ }^{15,18}$ Compared to HOMA-IR, the TyG index does not require quantification of insulin but does require FPB and fasting TG. It is well known that an increased TyG index is associated with increased risks of T2DM and CVD. ${ }^{18-21}$ Recently, several studies have found a significant correlation between the TyG index and clinical prognosis in patients with ACS. Zhao et al found that the TyG index may be a better predictor of cardiovascular risk than FPG or HbA1C for patients with ACS undergoing PCI. ${ }^{22} \mathrm{Hu}$ et al found that the TyG index may be a better predictor of cardiovascular risk than FPG or HbA1C for patients with ACS undergoing PCI. ${ }^{23}$ Luo et al also found that an increased TyG index may be an effective indicator of worse prognosis in patients with acute ST-segment elevation myocardial infarction (STEMI) who were treated with PCI for a 1-year follow-up. ${ }^{24}$ To date, there 
A

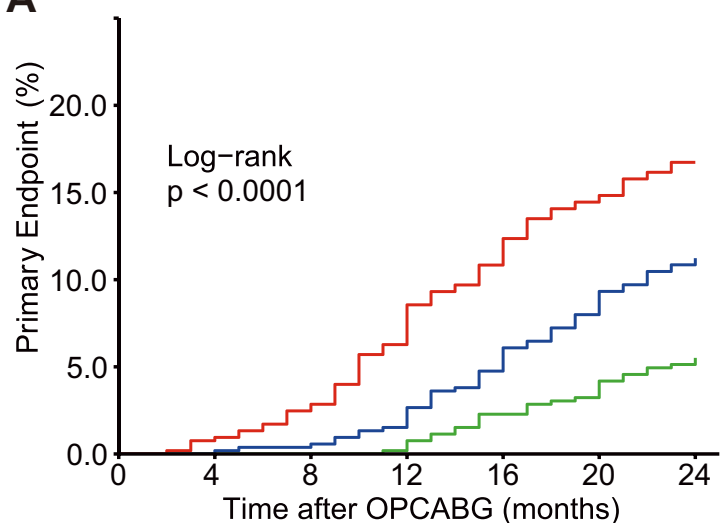

Num. at risk

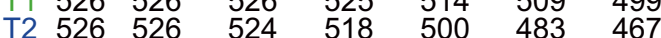

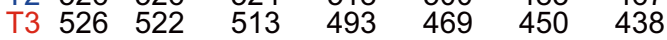

C

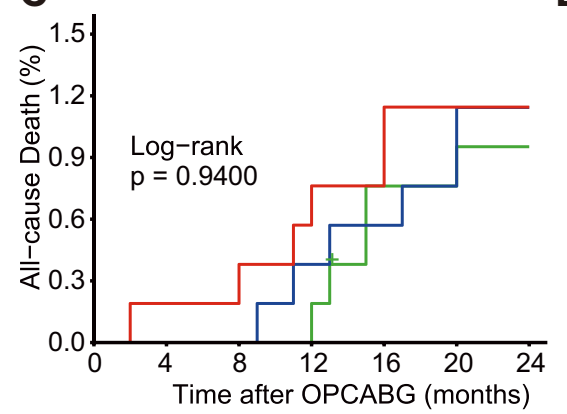

Num. at risk

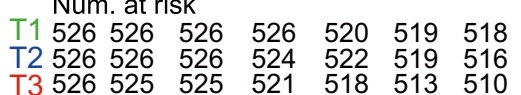

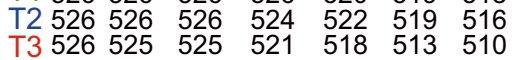

TyG Index

D

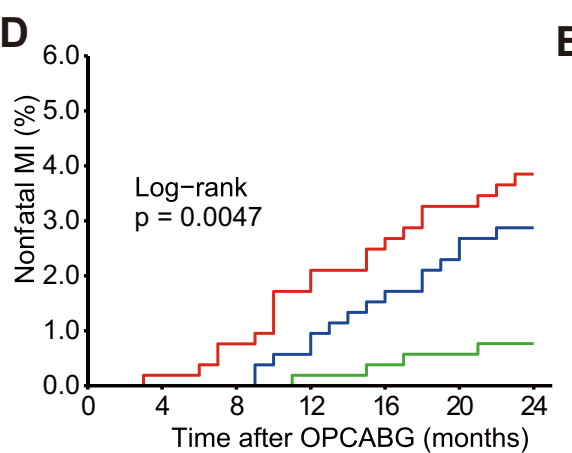

Num. at risk

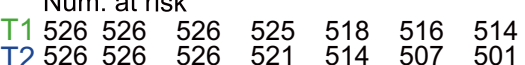

$\begin{array}{lllllll}\text { T2 } 526 & 526 & 526 & 521 & 514 & 507 & 501 \\ \text { T3 } 526 & 524 & 521 & 512 & 505 & 496 & 490\end{array}$
B

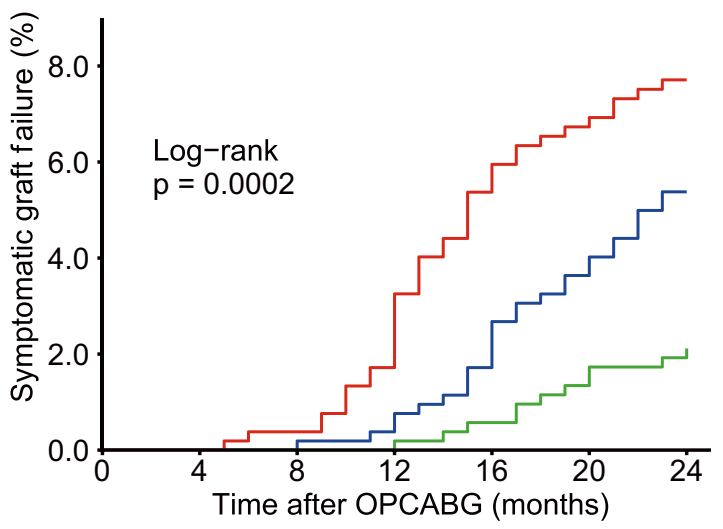

Num. at risk

$\begin{array}{lllllll}\text { T1 } 526 & 526 & 526 & 526 & 516 & 511 & 507 \\ \text { T2 } 526 & 526 & 526 & 522 & 513 & 500 & 488 \\ \text { T3 } 526 & 525 & 523 & 512 & 490 & 478 & 469\end{array}$

E

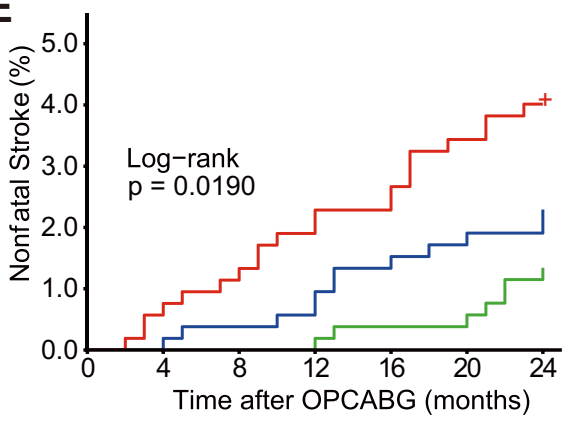

Num. at risk

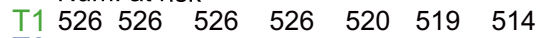

T2 $526 \quad 526 \quad 524 \quad 521 \quad 516 \quad 513 \quad 510$

T3 $526 \quad 523 \quad 520 \quad 513 \quad 511 \quad 502 \quad 499$

Figure 2 The TyG index and risk: Kaplan-Meier curves for the incidences of the primary endpoint (A), symptomatic graft failure (B), all-cause death (C), non-fatal MI (D), and non-fatal stroke (E) among the three study groups based on the TyG index tertiles.

Abbreviations: MI, myocardial infarction; OPCABG, off-pump coronary artery bypass graft.

are no reported studies on the TyG index and CABG to the best of our knowledge. We extend previous studies by proposing that the TyG index might be an effective indicator of the adverse prognosis of OPCABG in patients with T2DM.

Whether the TyG index is able to predict OPCABG outcomes in patients with established T2DM remains unknown. To our knowledge, our study first proposed the association between the TyG index and long-term MACCE of patients with diabetes underwent OPCABG. Moreover, our study is the first to take all-cause death, nonfatal MI, nonfatal stroke and symptomatic graft failure as the composite endpoint events. Our study demonstrated that a higher TyG index was significantly associated with a higher risk of MACCE. The higher risk of MACCE persisted after adjusting for traditional cardiovascular risk factors, burden of comorbidities. Thus, our study supports previous studies showing an association between the TyG index and adverse cardiovascular events in CAD patients.

We also found that after adjusting for important variables, the TyG index remained independently predictive of symptomatic graft failure irrespective of the use of arterial grafts. This means IR may affect both artery and vein grafts. The TyG index was reported to be positively associated with arterial stiffness as assessed by brachial-ankle or carotidfemoral pulse wave velocity. ${ }^{25-27}$ As the TyG index includes triglycerides and glucose in its formula and fasting triglycerides and glucose were shown to be associated with both long-term and short-term cardiovascular risk of patients with ACS, independent of diabetic status, ${ }^{28,29}$ the relationships between the TyG index and adverse cardiovascular 
Table 3 Relationship Between the Incidence of the Primary Endpoint and the TyG Index Expressed as a Continuous Variable

\begin{tabular}{|c|c|c|c|c|}
\hline Variables & $\begin{array}{l}\text { Univariate Analysis } \\
\text { HR }(95 \% \mathrm{Cl})\end{array}$ & $P$ value & $\begin{array}{l}\text { Multivariate } \\
\text { Analysis HR } \\
(95 \% \mathrm{Cl})\end{array}$ & $P$ value \\
\hline TyG index & $2.624(2.076-3.316)$ & $<0.001$ & $1.954(1.508-2.533)$ & $<0.001$ \\
\hline Age & $1.018(0.999-1.037)$ & 0.065 & $1.013(0.992-1.034)$ & 0.224 \\
\hline Sex, male & $0.729(0.536-0.993)$ & 0.045 & $0.766(0.556-1.055)$ & 0.103 \\
\hline BMI & I.07। (I.024-I.I2I) & 0.003 & $1.039(0.991-1.09)$ & 0.116 \\
\hline Current smoking & I.048 (0.758-I.448) & 0.778 & $0.933(0.668-1.305)$ & 0.686 \\
\hline Hypertension & I.084 (0.776-I.5।4) & 0.636 & I.I2 (0.79I-I.587) & 0.523 \\
\hline Previous MI & $1.416(0.970-2.067)$ & 0.072 & $1.322(0.887-|.97|)$ & 0.170 \\
\hline Previous stroke & $1.936(1.293-2.900)$ & 0.001 & $1.643(1.084-2.492)$ & 0.019 \\
\hline Past PCI & $1.087(0.773-1.529)$ & 0.631 & $0.97(0.68 I-1.382)$ & 0.866 \\
\hline Cardiac failure & $1.850(1.005-3.406)$ & 0.048 & $2.016(0.87 I-4.666)$ & 0.102 \\
\hline CKD & $1.129(0.556-2.295)$ & 0.737 & $0.832(0.403-1.72)$ & 0.620 \\
\hline Preoperative LVEF & $0.994(0.975-1.013)$ & 0.521 & $1.011(0.987-1.035)$ & 0.372 \\
\hline Insulin dependence & $1.314(0.96 \mathrm{I}-1.798)$ & 0.087 & $1.168(0.849-1.607)$ & 0.340 \\
\hline LDL-C & $1.005(1.001-1.01)$ & 0.016 & $1.003(0.998-1.007)$ & 0.244 \\
\hline HDL-C & $0.952(0.933-0.97)$ & $<0.001$ & $0.973(0.953-0.995)$ & 0.014 \\
\hline \multicolumn{5}{|l|}{ Diagnosis } \\
\hline Stable CAD & Ref. & & Ref. & \\
\hline UA & $0.611(0.085-4.364)$ & 0.623 & $0.421(0.055-3.247)$ & 0.407 \\
\hline STEMI & $0.608(0.076-4.858)$ & 0.639 & $0.359(0.042-3.083)$ & 0.350 \\
\hline NSTEMI & $4.208(0.57|-3| .0 \mid 4)$ & 0.159 & $2.167(0.274-17.119)$ & 0.463 \\
\hline \multicolumn{5}{|l|}{ Extent of CAD } \\
\hline I-vessel disease & Ref. & & Ref. & \\
\hline 2-vessel disease & $0.611(0.085-4.364)$ & 0.623 & $0.727(0.3 \mid 5-1.678)$ & 0.455 \\
\hline 3-vessel disease & $0.608(0.076-4.858)$ & 0.639 & $1.427(0.718-2.838)$ & 0.310 \\
\hline Left main disease & $4.208(0.57|-3| .0 \mid 4)$ & 0.159 & $0.64 \mid(0.38-1.082)$ & 0.096 \\
\hline Use of arterial graft & $\mathrm{I} .483(0.759-2.90 \mathrm{I})$ & 0.249 & $\mathrm{I} .042(0.733-|.48|)$ & 0.819 \\
\hline Complete & $0.505(0.28 I-0.908)$ & 0.022 & $0.327(0.176-0.61)$ & $<0.001$ \\
\hline \multicolumn{5}{|l|}{ revascularization } \\
\hline Use of IABP & $\mathrm{I} .483(0.759-2.90 \mathrm{I})$ & 0.249 & $1.429(0.695-2.94)$ & 0.332 \\
\hline
\end{tabular}

Abbreviations: TyG, triglyceride glucose; BMI, body mass index; MI, myocardial infarction; PCl, percutaneous coronary intervention; CKD, chronic kidney disease; LVEF, left ventricular ejection fraction; LDL-C, low-density lipoprotein-cholesterol; HDL-C, high-density lipoprotein-cholesterol; CAD, coronary artery disease; UA, unstable angina; STEMI, ST-segment elevation myocardial infarction; NSTEMI, non ST-segment elevation myocardial infarction; IABP, intra-aortic balloon pump.

outcomes in diabetic patients might be in part explained. Moreover, we found that BMI and LDL-C levels were significantly higher in patients in the highest TyG index tertile than in those in other tertiles, whereas HDL-C levels were lower. Therefore, the association of the TyG index with adverse cardiovascular outcomes may be partially mediated by these traditional cardiovascular risk factors.

The potential mechanism in the association of the TyG index with the development and progression of cardiovascular disease remains uncertain. As a reliable marker of the severity of IR, proatherogenic properties of IR may partly account for the association. ${ }^{30,31}$ In this study, patients with a high TyG index were more likely to have higher BMI, LDL-C, and have lower HDL-C, suggesting that the observed association between the TyG index and poor prognosis may be explained by the presence of cardiovascular risk factors. IR can induce an imbalance in glucose metabolism that results in chronic hyperglycemia, which leads to oxidative stress and inflammatory response that triggers vascular endothelial cell injury. IR can also affect lipid metabolism, which then leads to the development of dyslipidemia and the well-known lipid triad: elevated plasma triglycerides, reduced plasma HDL-C, and the appearance of small dense LDL-C particles. These metabolic changes contribute to atherosclerotic plaque formation. ${ }^{32} \mathrm{~A}$ higher TyG index may affect native 
Table 4 Relationship Between the Incidence of the Primary Endpoint and the TyG Index Expressed as a Categorical Variable

\begin{tabular}{|c|c|c|c|c|}
\hline Variables & $\begin{array}{l}\text { Univariate Analysis } \\
\text { HR }(95 \% \mathrm{Cl})\end{array}$ & $P$ value & $\begin{array}{l}\text { Multivariate } \\
\text { Analysis } \\
\text { HR }(95 \% \mathrm{Cl})\end{array}$ & $P$ value \\
\hline \multicolumn{5}{|l|}{ TyG index tertiles } \\
\hline TI & Ref. & & Ref. & \\
\hline T2 & $2.095(1.343-3.268)$ & 0.001 & $1.726(1.093-2.725)$ & 0.019 \\
\hline T3 & $3.297(2.167-5.016)$ & $<0.001$ & $2.133(1.347-3.377)$ & 0.001 \\
\hline Age & $1.018(0.999-1.037)$ & 0.065 & $1.016(0.995-1.038)$ & 0.132 \\
\hline Sex, male & $0.729(0.536-0.993)$ & 0.045 & $0.755(0.549-1.04)$ & 0.085 \\
\hline BMI & $1.07 \mid(1.024-1.121)$ & 0.003 & $1.045(0.996-1.098)$ & 0.072 \\
\hline Current smoking & $1.048(0.758-1.448)$ & 0.778 & $0.963(0.69-1.344)$ & 0.823 \\
\hline Hypertension & $1.084(0.776-|.5| 4)$ & 0.636 & $1.075(0.76-1.522)$ & 0.683 \\
\hline Previous MI & $1.416(0.970-2.067)$ & 0.072 & $1.299(0.87 \mid-1.938)$ & 0.200 \\
\hline Previous stroke & $1.936(1.293-2.900)$ & 0.001 & $1.624(1.07 I-2.465)$ & 0.023 \\
\hline Past PCI & $1.087(0.773-1.529)$ & 0.631 & $0.96(0.674-1.367)$ & 0.821 \\
\hline Cardiac failure & $1.850(1.005-3.406)$ & 0.048 & $2.162(0.933-5.009)$ & 0.072 \\
\hline CKD & I.I $29(0.556-2.295)$ & 0.737 & $0.86 I(0.416-1.783)$ & 0.687 \\
\hline Preoperative LVEF & $0.994(0.975-1.013)$ & 0.521 & $1.009(0.986-1.034)$ & 0.447 \\
\hline Insulin dependence & $1.314(0.96 \mathrm{I}-1.798)$ & 0.087 & $1.189(0.866-1.634)$ & 0.284 \\
\hline LDL-C (mg/dl) & $1.005(1.001-1.01)$ & 0.016 & $1.003(0.999-1.008)$ & 0.176 \\
\hline HDL-C (mg/dl) & $0.952(0.933-0.97)$ & $<0.001$ & $0.966(0.946-0.988)$ & 0.002 \\
\hline \multicolumn{5}{|l|}{ Diagnosis } \\
\hline Stable CAD & Ref. & & Ref. & \\
\hline UA & $0.6 \mathrm{II}(0.085-4.364)$ & 0.623 & $0.467(0.06-3.604)$ & 0.465 \\
\hline STEMI & $0.608(0.076-4.858)$ & 0.639 & $0.416(0.048-3.572)$ & 0.424 \\
\hline NSTEMI & $4.208(0.57|-31.0| 4)$ & 0.159 & $2.689(0.34-21.246)$ & 0.348 \\
\hline \multicolumn{5}{|l|}{ Extent of CAD } \\
\hline I-vessel disease & Ref. & & Ref. & \\
\hline 2-vessel disease & $0.611(0.085-4.364)$ & 0.623 & $0.686(0.297-1.586)$ & 0.379 \\
\hline 3-vessel disease & $0.608(0.076-4.858)$ & 0.639 & $1.4 \mathrm{II}(0.709-2.807)$ & 0.327 \\
\hline Left main disease & $4.208(0.57|-31.0| 4)$ & 0.159 & $0.634(0.376-1.07)$ & 0.088 \\
\hline Use of arterial graft & $1.483(0.759-2.901)$ & 0.249 & I.035 (0.729-I.47) & 0.846 \\
\hline $\begin{array}{l}\text { Complete } \\
\text { revascularization }\end{array}$ & $0.505(0.28 I-0.908)$ & 0.022 & $0.306(0.163-0.574)$ & $<0.001$ \\
\hline Use of IABP & $\mathrm{I} .483(0.759-2.901)$ & 0.249 & $1.397(0.675-2.893)$ & 0.368 \\
\hline
\end{tabular}

Abbreviations: TyG, triglyceride glucose; BMI, body mass index; MI, myocardial infarction; PCl, percutaneous coronary intervention; CKD, chronic kidney disease; LVEF, left ventricular ejection fraction; LDL-C, low-density lipoprotein-cholesterol; HDL-C, high-density lipoprotein-cholesterol; CAD, coronary artery disease; UA, unstable angina; STEMI, ST-segment elevation myocardial infarction; NSTEMI, non ST-segment elevation myocardial infarction; IABP, intra-aortic balloon pump.

coronary atherosclerosis because the TyG index has been demonstrated to be related to coronary artery calcification and arterial stiffness. ${ }^{26,33-35}$ In addition, IR is associated with coronary plaque vulnerability. ${ }^{35,36}$ In contrast, a higher level of TyG may accelerate the atherosclerosis of grafts and accelerate the process of graft failure because IR has a proinflammatory and procoagulatory effect and is associated with endothelial dysfunction. ${ }^{37,38}$ Consistent with previous studies, the TyG index was positively associated with the severity of CAD, suggesting that a difference in the extent of coronary atherosclerosis may contribute to the graded TyG index-MACCE relationship. ${ }^{39,40}$ Therefore, the TyG index may reflect IR from these two aspects and thus be closely related to IR, which has been widely demonstrated to have a significant relationship with endothelial dysfunction, oxidative stress, cardiovascular remodeling, coagulation imbalance and inflammatory response, leading to late graft failure. More efforts must be made to elucidate the exact mechanism of the association between the TyG index and CVEs. 
This study had several limitations. 1) The study was a single-center, retrospective, observational study in a highly selected cohort with strict exclusion criteria, and the sample size was relatively small, which may have weakened the power of the results. Further retrospective, multicenter studies in a more extensive population with a larger sample size are needed to verify the present findings. 2) The TyG index was assessed only once at admission. The changes in the TyG index during the follow-up period, which may have better predictive value for adverse prognosis, were not assessed in our study. 3) A certain proportion of participants received lipid-regulating therapy and antidiabetic treatment at admission, which may have had a potential impact on the TyG index and the study results. 4) Nearly all of the study population was Chinese patients. The results should be cautiously interpreted and expanded to Western populations as differences in metabolic levels exist among different races. 5) Although we took insulin into consideration, the other medicine therapy had not been considered, which may have had a potential impact on the study results. 6) It is difficult to rule out that some patients may be complicated with undiagnosed systemic diseases, such as occult malignancies, which may have an impact on the assessment of prognosis.

\section{Conclusions}

Based on retrospective studies, we concluded that the TyG index is associated with 2-year MACCEs in patients with T2DM who underwent OPCABG independently of known cardiovascular risk factors. The TyG index may be a useful marker for prognosis in patients with T2DM who underwent OPCABG. Further prospective, large studies are required to confirm our findings.

\section{Data Sharing Statement}

The datasets used during the current study are available from the corresponding author on reasonable request.

\section{Ethics Approval and Consent to Participate}

This study was approved by the institutional review board of Beijing Anzhen Hospital, Capital Medical University. Given the retrospective nature of this study, the requirement for informed consent was waived. All patients' identifiable information were hidden, and anyone's identity cannot be deduced from the context.

\section{Author Contributions}

All authors made substantial contributions to conception and design, acquisition of data, or analysis and interpretation of data; took part in drafting the article or revising it critically for important intellectual content; agreed to submit to the current journal; gave final approval of the version to be published; and agree to be accountable for all aspects of the work.

\section{Funding}

This work was supported by the Capital Health Research and Development of Special Fund (2020-1-2061).

\section{Disclosure}

The authors declare that the research was conducted in the absence of any commercial or financial relationships that could be construed as a potential conflict of interest.

\section{References}

1. Neumann FJ, Sousa-Uva M, Ahlsson A, et al. 2018 ESC/EACTS guidelines on myocardial revascularization. Eur Heart J. 2019;40(2):87-165. doi:10.1093/eurheartj/ehy394

2. Zhou P, Zhu P, Xiao Z, et al. Meta-analysis of repeat revascularization of off-pump and on-pump coronary artery bypass surgery. Ann Thorac Surg. 2018;106(2):526-531. doi:10.1016/j.athoracsur.2018.02.068

3. Pevni D, Ben-Gal Y, Mohr R, et al. One or two internal thoracic grafts? Long-term follow-up of 957 off-pump coronary bypass surgeries. Ann Thorac Surg. 2017;104(1):70-77. doi:10.1016/j.athoracsur.2016.10.049

4. Du T, Yuan G, Zhang M, et al. Clinical usefulness of lipid ratios, visceral adiposity indicators, and the triglycerides and glucose index as risk markers of insulin resistance. Cardiovasc Diabetol. 2014;13:146. doi:10.1186/s12933-014-0146-3

5. Simental-Mendía LE, Rodríguez-Morán M, Guerrero-Romero F. The product of fasting glucose and triglycerides as surrogate for identifying insulin resistance in apparently healthy subjects. Metab Syndr Relat Disord. 2008;6(4):299-304. doi:10.1089/met.2008.0034 
6. Xie Y, Guo R, Li Z, et al. Temporal relationship between body mass index and triglyceride-glucose index and its impact on the incident of hypertension. Nutr Metab Cardiovasc Dis. 2019;29(11):1220-1229. doi:10.1016/j.numecd.2019.07.003

7. Liu EQ, Weng YP, Zhou AM, et al. Association between triglyceride-glucose index and type 2 diabetes mellitus in the Japanese population: a secondary analysis of a retrospective cohort study. Biomed Res Int. 2020;2020:2947067. doi:10.1155/2020/2947067

8. Low S, Khoo KCJ, Irwan B, et al. The role of triglyceride glucose index in development of type 2 diabetes mellitus. Diabetes Res Clin Pract. 2018;143:43-49. doi:10.1016/j.diabres.2018.06.006

9. Ma X, Dong L, Shao Q, et al. Triglyceride glucose index for predicting cardiovascular outcomes after percutaneous coronary intervention in patients with type 2 diabetes mellitus and acute coronary syndrome. Cardiovasc Diabetol. 2020;19(1):31. doi:10.1186/s12933-020-01006-7

10. Chiu TH, Tsai HJ, Chiou HC, et al. A high triglyceride-glucose index is associated with left ventricular dysfunction and atherosclerosis. Int $J$ Med Sci. 2021;18(4):1051-1057. doi:10.7150/ijms.53920

11. Lambrinoudaki I, Kazani MV, Armeni E, et al. The TyG index as a marker of subclinical atherosclerosis and arterial stiffness in lean and overweight postmenopausal women. Heart Lung Circ. 2018;27(6):716-724. doi:10.1016/j.hlc.2017.05.142

12. Alizargar J, Bai CH, Hsieh NC, et al. Use of the triglyceride-glucose index (TyG) in cardiovascular disease patients. Cardiovasc Diabetol. 2020;19 (1):8. doi:10.1186/s12933-019-0982-2

13. Cockcroft DW, Gault MH. Prediction of creatinine clearance from serum creatinine. Nephron. 1976;16(1):31-41. doi:10.1159/000180580

14. Nam KW, Kwon HM, Jeong HY, et al. High triglyceride-glucose index is associated with subclinical cerebral small vessel disease in a healthy population: a cross-sectional study. Cardiovasc Diabetol. 2020;19(1):53. doi:10.1186/s12933-020-01031-6

15. Vasques AC, Novaes FS, de Oliveira Mda S, et al. TyG index performs better than HOMA in a Brazilian population: a hyperglycemic clamp validated study. Diabetes Res Clin Pract. 2011;93(3):e98-e100. doi:10.1016/j.diabres.2011.05.030

16. Irace C, Carallo C, Scavelli FB, et al. Markers of insulin resistance and carotid atherosclerosis. A comparison of the homeostasis model assessment and triglyceride glucose index. Int J Clin Pract. 2013;67(7):665-672. doi:10.1111/ijcp.12124

17. Wallace TM, Matthews DR. The assessment of insulin resistance in man. Diabet Med. 2002;19(7):527-534. doi:10.1046/j.1464-5491.2002.00745.x

18. Lee SH, Kwon HS, Park YM, et al. Predicting the development of diabetes using the product of triglycerides and glucose: the Chungju Metabolic Disease Cohort (CMC) study. PLoS One. 2014;9(2):e90430. doi:10.1371/journal.pone.0090430

19. Navarro-González D, Sánchez-íñigo L, Pastrana-Delgado J, et al. Triglyceride-glucose index (TyG index) in comparison with fasting plasma glucose improved diabetes prediction in patients with normal fasting glucose: the vascular-metabolic CUN cohort. Prev Med. 2016;86:99-105. doi:10.1016/j.ypmed.2016.01.022

20. Sánchez-ínigo L, Navarro-González D, Fernández-Montero A, et al. The TyG index may predict the development of cardiovascular events. Eur $J$ Clin Invest. 2016;46(2):189-197. doi:10.1111/eci.12583

21. Li S, Guo B, Chen H, et al. The role of the triglyceride (triacylglycerol) glucose index in the development of cardiovascular events: a retrospective cohort analysis. Sci Rep. 2019;9(1):7320. doi:10.1038/s41598-019-43776-5

22. Zhao Q, Zhang TY, Cheng YJ, et al. Impacts of triglyceride-glucose index on prognosis of patients with type 2 diabetes mellitus and non-STsegment elevation acute coronary syndrome: results from an observational cohort study in China. Cardiovasc Diabetol. 2020;19(1):108. doi:10.1186/s12933-020-01086-5

23. Hu C, Zhang J, Liu J, et al. Discordance between the triglyceride glucose index and fasting plasma glucose or HbA1C in patients with acute coronary syndrome undergoing percutaneous coronary intervention predicts cardiovascular events: a cohort study from China. Cardiovasc Diabetol. 2020;19(1):116. doi:10.1186/s12933-020-01091-8

24. Luo E, Wang D, Yan G, et al. High triglyceride-glucose index is associated with poor prognosis in patients with acute ST-elevation myocardial infarction after percutaneous coronary intervention. Cardiovasc Diabetol. 2019;18(1):150. doi:10.1186/s12933-019-0957-3

25. Lee SB, Ahn CW, Lee BK, et al. Association between triglyceride glucose index and arterial stiffness in Korean adults. Cardiovasc Diabetol. 2018;17(1):41. doi:10.1186/s12933-018-0692-1

26. Zhao S, Yu S, Chi C, et al. Association between macro- and microvascular damage and the triglyceride glucose index in community-dwelling elderly individuals: the Northern Shanghai Study. Cardiovasc Diabetol. 2019;18(1):95. doi:10.1186/s12933-019-0898-x

27. Levisianou D, Foussas S, Skopelitis E, et al. Arterial stiffness predicts risk for long-term recurrence in patients with type 2 diabetes admitted for acute coronary event. Diabetes Res Clin Pract. 2013;99(3):315-320. doi:10.1016/j.diabres.2012.11.023

28. Schwartz GG, Abt M, Bao W, et al. Fasting triglycerides predict recurrent ischemic events in patients with acute coronary syndrome treated with statins. J Am Coll Cardiol. 2015;65(21):2267-2275. doi:10.1016/j.jacc.2015.03.544

29. Sinnaeve PR, Steg PG, Fox KA, et al. Association of elevated fasting glucose with increased short-term and 6-month mortality in ST-segment elevation and non-ST-segment elevation acute coronary syndromes: the global registry of acute coronary events. Arch Intern Med. 2009;169 (4):402-409. doi:10.1001/archinternmed.2008.572

30. Iguchi $\mathrm{T}$, Hasegawa $\mathrm{T}$, Otsuka $\mathrm{K}$, et al. Insulin resistance is associated with coronary plaque vulnerability: insight from optical coherence tomography analysis. Eur Heart J Cardiovasc Imaging. 2014;15(3):284-291. doi:10.1093/ehjci/jet158

31. An X, Yu D, Zhang R, et al. Insulin resistance predicts progression of de novo atherosclerotic plaques in patients with coronary heart disease: a one-year follow-up study. Cardiovasc Diabetol. 2012;11:71. doi:10.1186/1475-2840-11-71

32. Ormazabal V, Nair S, Elfeky O, et al. Association between insulin resistance and the development of cardiovascular disease. Cardiovasc Diabetol. 2018;17(1):122. doi:10.1186/s12933-018-0762-4

33. Won KB, Park EJ, Han D, et al. Triglyceride glucose index is an independent predictor for the progression of coronary artery calcification in the absence of heavy coronary artery calcification at baseline. Cardiovasc Diabetol. 2020;19(1):34. doi:10.1186/s12933-020-01008-5

34. Fiorentino TV, Marini MA, Succurro E, et al. Relationships of surrogate indexes of insulin resistance with insulin sensitivity assessed by euglycemic hyperinsulinemic clamp and subclinical vascular damage. BMJ Open Diabetes Res Care. 2019;7(1):e000911. doi:10.1136/bmjdrc2019-000911

35. Nakagomi A, Sunami Y, Kawasaki Y, et al. Sex difference in the association between surrogate markers of insulin resistance and arterial stiffness. J Diabetes Complications. 2020;34(6):107442. doi:10.1016/j.jdiacomp.2019.107442

36. Mitsuhashi T, Hibi K, Kosuge M, et al. Relation between hyperinsulinemia and nonculprit plaque characteristics in nondiabetic patients with acute coronary syndromes. JACC Cardiovasc Imaging. 2011;4(4):392-401. doi:10.1016/j.jcmg.2011.02.004 
37. Virdis A, Santini F, Colucci R, et al. Vascular generation of tumor necrosis factor- $\alpha$ reduces nitric oxide availability in small arteries from visceral fat of obese patients. J Am Coll Cardiol. 2011;58(3):238-247. doi:10.1016/j.jacc.2011.01.050

38. Plomgaard P, Bouzakri K, Krogh-Madsen R, et al. Tumor necrosis factor-alpha induces skeletal muscle insulin resistance in healthy human subjects via inhibition of Akt substrate 160 phosphorylation. Diabetes. 2005;54(10):2939-2945. doi:10.2337/diabetes.54.10.2939

39. Mao Q, Zhou D, Li Y, et al. The triglyceride-glucose index predicts coronary artery disease severity and cardiovascular outcomes in patients with non-ST-segment elevation acute coronary syndrome. Dis Markers. 2019;2019:6891537. doi:10.1155/2019/6891537

40. Lee EY, Yang HK, Lee J, et al. Triglyceride glucose index, a marker of insulin resistance, is associated with coronary artery stenosis in asymptomatic subjects with type 2 diabetes. Lipids Health Dis. 2016;15(1):155. doi:10.1186/s12944-016-0324-2

Diabetes, Metabolic Syndrome and Obesity: Targets and Therapy

\section{Publish your work in this journal}

Diabetes, Metabolic Syndrome and Obesity: Targets and Therapy is an international, peer-reviewed open-access journal committed to the rapid publication of the latest laboratory and clinical findings in the fields of diabetes, metabolic syndrome and obesity research. Original research, review, case reports, hypothesis formation, expert opinion and commentaries are all considered for publication. The manuscript management system is completely online and includes a very quick and fair peer-review system, which is all easy to use. Visit http://www.dovepress. com/testimonials.php to read real quotes from published authors.

Submit your manuscript here: https://www.dovepress.com/diabetes-metabolic-syndrome-and-obesity-targets-and-therapy-journal 